\title{
MELodica Orchestra for DYspnea (MELODY): A Randomized Safety, Feasibility, and Efficacy Pilot
}

Mackenzie McGrath ${ }^{1}$, Debra S. Burns ${ }^{8}$, Shannon Crow $^{2}$, Aimee Lillie $2-3$, Laura J. Myers $^{2-4}$, Jennifer Myers ${ }^{2-3}$, Anthony J. Perkins ${ }^{2,7}$, Nicholas Rattray ${ }^{2-3,9}$, Joseph Smith $^{1,11-12}$, Sally Wasmuth ${ }^{10}$, Dawn M. Bravata ${ }^{2-6}$

${ }^{1}$ Indiana University School of Medicine, Indianapolis, IN, ${ }^{2}$ Department of Veterans Affairs (VA) Health Services Research and Development (HSR\&D) Precision Monitoring to Transform Care (PRISM) Quality Enhancement Research Initiative (QUERI), ${ }^{3}$ VA HSR\&D Center for Health Information and Communication (CHIC), Richard L. Roudebush VA Medical Center, Indianapolis,

IN, ${ }^{4}$ Department of Internal Medicine, Indiana University School of Medicine, Indianapolis, IN,

${ }^{5}$ Department of Neurology, Indiana University School of Medicine, Indianapolis, IN, ${ }^{6}$ Regenstrief Institute, Indianapolis, IN, ${ }^{7}$ Department of Biostatistics, Indiana University School of Medicine, IUPUI, Indianapolis, IN, ${ }^{8}$ Department of Music and Arts Technology, IUPUI, Indianapolis, IN,

${ }^{9}$ Department of Anthropology, IUPUI, Indianapolis, IN, ${ }^{10}$ School of Occupational Therapy,

University of Indianapolis, Indianapolis, IN, ${ }^{11}$ Department of Medicine Division of Pulmonary, Critical Care, Sleep \& Occupational Medicine, Indiana University School of Medicine, Indianapolis, IN, ${ }^{12}$ Richard L. Roudebush VA Medical Center

\section{Background}

Chronic obstructive pulmonary disease (COPD) is highly prevalent among Veterans. Patients with COPD commonly experience dyspnea, which can be debilitating and may limit daily activities leading to a reduced quality of life. Pulmonary rehabilitation often includes teaching pursed lip breathing as a method to reduce dyspnea. However, patients with COPD, including Veterans, have limited access to pulmonary rehabilitation due to transportation constraints and lack of referrals by physicians.

\section{Methods}

MELodica Orchestra for DYspnea (MELODY) is a randomized controlled clinical trial designed to assess the safety, feasibility, and efficacy of a music-based approach to teach pursed lip breathing to Veterans with COPD experiencing dyspnea. Patients will be randomized in a 1:1:1 ratio to the intervention group, education only control group, and usual care control group using block randomization. Patients in the intervention group will meet twice weekly over twelve weeks for instruction on how to play the melodica and to participate in group music-making. The program also includes education about COPD, pursed lip breathing, tobacco cessation, and other relevant topics. Each participant will receive quantitative assessments (e.g., exercise endurance and dyspnea scales) and participate in qualitative interviews.

\section{Anticipated Results}

We expect the intervention to be safe and feasible. We hypothesize that patients in the intervention group will achieve the greatest reduction in dyspnea compared with patients in either control group.

\section{Potential Impact}

If pilot data demonstrates efficacy, then a multiple-site randomized control trial will be conducted with intent to introduce the program into routine clinical practice. 